\title{
Wissen von Hausärzten und Ärzten in Weiterbildung über nationale Asthmaleitlinien
}

\author{
Asthma Guidelines: Knowledge of Family Doctors and Trainees in Germany
}

Autoren

Institute
H. Lingner ${ }^{1}$, D. Piepenschneider ${ }^{1}$, T. Schleef ${ }^{1}$, C.-P. Criée ${ }^{2}$, P. Kardos ${ }^{3}$, H. Worth ${ }^{4}$, E. Hummers-Pradier ${ }^{5}$

Die Institutsangaben sind am Ende des Beitrags gelistet. eingereicht 2.4.2013

akzeptiert $\quad 4.4 .2013$

\section{Bibliografie}

Dol http://dx.doi.org/

$10.1055 / \mathrm{s}-0033-1344033$

Online-Publikation: 22.5.2013

Pneumologie 2013; 67: 335-339

(c) Georg Thieme Verlag KG

Stuttgart · New York

ISSN 0934-8387

\section{Korrespondenzadresse \\ Prof. Dr. med. \\ Eva Hummers-Pradier}

Institut für Allgemeinmedizin, Universitätsmedizin Göttingen

Georg-August-Universität

Humboldtallee 38

37073 Göttingen

Eva.Hummers-Pradier@med.uni-

goettingen.de

\section{Zusammenfassung \\ $\nabla$}

In Deutschland und international wurden evidenzbasierte Leitlinien als Entscheidungshilfe bei Diagnostik und Therapie des Asthma bronchiale entwickelt, deren Empfehlungen allerdings oft nur teilweise umgesetzt werden. Ein Projekt der Autoren exploriert mögliche Ursachen: das Wissen um Leitlinieninhalte, die praxistaugliche Gestaltung von Leitlinien sowie Einstellungen von Ärzten und Patienten. Der vorliegende Beitrag vergleicht Ergebnisse einer Wissensbefragung bei Hausärzten und Ärzten in Weiterbildung in der Allgemeinmedizin mit ähnlichen national und international publizierten Untersuchungen. Es zeigten sich in allen Studien und bei allen untersuchten Arztgruppen deutliche Wissensdefizite. Viele der in der eigenen Studie befragten Ärzte präferierten irrtümlich die nicht bzw. nur in Ausnahmefällen indizierten Mukolytika und Antibiotika bei der Behandlung von Asthmapatienten mit akuten oder subakuten Symptomen und verkennen teilweise deren entzündlichen Charakter und die wichtige Rolle inhalativer (und auch systemischer) Kortikosteroide bei der Dauertherapie des Asthmas (bzw. akuter Verschlechterungen).

Asthma bronchiale ist mit einer Prävalenz von 5-7\% eine häufige chronische Erkrankung in Deutschland [1]. Patienten mit asthmatischen Beschwerden konsultieren meist initial einen Hausarzt, dem dadurch eine Schlüsselposition bei der Diagnostik und Therapie zukommt. Um ärztliche Entscheidungen zu unterstützen und eine optimale Versorgung der Patienten zu gewährleisten, wurden Leitlinien wie die Leitlinie der Deutschen Atemwegsliga und der Deutschen Gesellschaft für Pneumologie und Beatmungsmedizin [2] sowie die Nationale VersorgungsLeitlinie (NVL) Asthma [3] entwickelt. Allerdings gibt es Hinweise, dass trotz breiter Dissemina-

\section{Abstract \\ $\nabla$}

In Germany as well as internationally, evidencebased asthma guidelines were developed as a decision aid for diagnosis and therapy. However, adherence to recommendations is usually less than optimal. The authors explore potential implementation barriers: knowledge gaps, practical presentation of guidelines, and attitudes of doctors and patients. In this paper, we compare results of our knowledge survey of family doctors and trainees with results of similar national and international studies. In all studies, all surveyed groups of physicians revealed considerable knowledge gaps. Many physicians in our own study erroneously preferred mucolytics and antibiotics to treat acute or subacute asthma symptoms though these drugs are not or only exceptionally indicated. The inflammatory character of asthma often goes unrecognized, as well as the importance of inhaled (and systemic) corticosteroids in long term treatment (or treatment of exacerbations).

tion die Umsetzung der Leitlinienempfehlungen noch nicht zufriedenstellend ist [4].

\section{Leitlinien \\ $\nabla$}

Die Bundesärztekammer definiert Leitlinien als systematische Entscheidungshilfen, die Informationen zum angemessenen Vorgehen bei speziellen gesundheitlichen Problemstellungen liefern sollen. Durch eine praxisorientierte Aufbereitung der besten aktuell verfügbaren Evidenz verfolgen Leitlinien folgende Ziele [5]: 
- Sicherung und Verbesserung der medizinischen Versorgung

- Motivation zu wissenschaftlich begründeter und ökonomisch sowie individuell angemessener ärztlicher Vorgehensweise unter Berücksichtigung der Bedürfnisse und Einstellungen des Patienten

- Verminderung unerwünschter Qualitätsschwankungen in der Versorgung

- Information der Öffentlichkeit über notwendige und allgemein übliche ärztliche Maßnahmen bei speziellen Gesundheitsrisiken und Gesundheitsstörungen

- Vermeidung unnötiger und überholter medizinischer Maßnahmen

- kosteneffizienter Einsatz von Ressourcen im Gesundheitswesen

Darüber hinaus können Leitlinienempfehlungen als Grundlage für Qualitätsmanagement dienen, leitlinienbasierte Qualitätsindikatoren werden in Benchmarking-Prozessen zur Bewertung der Versorgungsqualität herangezogen [5]. Allerdings ist eine Umsetzung von Leitlinienempfehlungen nicht bei jedem Patienten möglich oder sinnvoll, sodass eine hundertprozentige Umsetzung auch bei guter Versorgungsqualität nicht zu erwarten ist und die für ein Benchmarking notwendige Kalibrierung meistens fehlt. Eine methodisch hochwertige Leitlinie muss international einheitlichen Kriterien gerecht werden, die in Deutschland im sogenannten Deutschen Leitlinienbewertungsinstrument (DELBI) zusammengefasst sind [6]. Zu den wichtigsten Aspekten zählen:

- Evidenzbasierung (systematische Recherche, Auswahl und Bewertung der Literatur)

- Repräsentativität des Leitliniengremiums (Einbezug von Vertretern aller relevanten Fach- bzw. Berufsgruppen sowie Ermittlung und Einbezug der Patientenperspektive)

- Strukturierte, transparente Konsensfindung (kritische Bewertung der Evidenzlage und Festlegung der Empfehlungen in einem formalen Prozess) $[5,6]$.

\section{Der Weg zu evidenzbasierten Asthmaleitlinien \\ $\nabla$}

In Deutschland veröffentlichte die 1979 gegründete Deutsche Atemwegsliga e.V. bereits 1980 „Empfehlungen zur Behandlung von akuten und chronischen Atemwegsobstruktionen mit Bronchospasmolytika in der Praxis“ [7]. Empfehlungen zur KortikoidTherapie folgten 1981, das erste Stufenschema zur Langzeittherapie 1984. Gegen Ende der 1990er entstand der Anspruch, dass Leitlinien evidenzbasiert sein sollen, statt lediglich Expertenmeinungen darzulegen [6]. Die erste umfassende Darstellung zur Asthmatherapie bei Kindern und Erwachsenen erschien 1998 [8]; deren Überarbeitung durch die Deutsche Atemwegsliga und die Deutsche Gesellschaft für Pneumologie und Beatmungsmedizin (DGP) wurde 2006 erstmals als Leitlinie bezeichnet [2]. 1993 initiierte das American National Heart, Lung and Blood Institute (NHLBI) in Kooperation mit der WHO die Global Initiative for Asthma (GINA), eine Organisation aus international führenden Asthma-Experten. GINA sollte eine weltweit einsetzbare Vorgehensweise zur Behandlung des Asthmas entwickeln und für die Verbreitung von Leitlinien inklusive deren Anpassung an die Gegebenheiten der jeweiligen Länder sorgen. Die erste Version der Global Strategy for Asthma Management and Prevention von 1995 [9] wurde in zahlreiche Sprachen übersetzt und diente als Grundlage für Asthma-Leitlinien auf der ganzen Welt [10]. Zu den international wichtigsten und einflussreichsten Asthma- leitlinien zählen heute die jährlich aktualisierte Leitlinie der British Thoracic Society (BTS) in Zusammenarbeit mit dem Scottish Intercollegiate Guidelines Network (SIGN) [11], der Expert Panel Report (EPR-3) des National Heart, Lung and Blood Institute (NHBLI) und der BTS von 2007 [12] sowie der zuletzt 2012 überarbeitete Global Strategy for Asthma Management and Prevention Report der GINA [13]. Die regelmäßige Aktualisierung der Nationalen Versorgungsleitlinie Asthma (NVL) [3] und der Leitlinie der Deutschen Atemwegsliga und der DGP [2] erfolgt unter Berücksichtigung der oben genannten internationalen Leitlinien.

\section{Implementierung von Leitlinien}

Um viele potenzielle Nutzer zu erreichen, bestehen hochwertige Leitlinien aus mehreren Modulen, u.a. Lang- und Kurzfassung sowie Patienteninformationen [5]. Allerdings hat sich im Laufe der letzten Jahre deutlich gezeigt, dass auch methodisch exzellente, weit verbreitete Leitlinien wenig positive Auswirkung auf das Verhalten von Ärzten erzielen, wenn nicht der „Transfer von Handlungsempfehlungen in individuelles Handeln bzw. in Verhaltensänderungen “ durch gezielte Implementierung unterstützt wird $[5,14,15]$. Erfolgreiche Implementierungsstrategien berücksichtigen immer spezifische strukurelle, organisatorische, personelle oder finanzielle Barrieren [5]. Wenig effektiv sind die passive Verbreitung von Leitlinien über das Internet, Publikationen in Fachzeitschriften sowie die Präsentation in Form von Frontalvorträgen $[5,16]$.

Folgende Maßnahmen versprechen einen größeren Erfolg [16]:

1. Eine Implementierung im Rahmen der Qualitätszirkelarbeit,

2. der Einbau von Leitlinieninhalten in das bestehende

Qualitätsmanagement,

3. die Integration von Erinnerungshilfen in die Praxis-/Krankenhaussoftware,

4. die Einführung von Checklisten für den behandelnden Arzt und/oder das Praxisteam,

5. eine regionale Leitlinienadaptierung

6. die Evaluation von Akzeptanzproblemen und Umsetzungsschwierigkeiten durch Befragung der Leitlinienanwender.

Welchen Erfolg die Implementierung im Rahmen von Disease Management Programmen (DMP) hat, muss im Einzelnen noch evaluiert werden. Allerdings enthalten DMPs die Erfolg versprechenden Elemente 2, 3 und 4 der obigen Liste.

\section{Untersuchungen zur Implementierung}

Trotz der Existenz hochwertiger Leitlinien sind viele Asthmapatienten suboptimal versorgt bzw. leiden unter einem schlecht kontrollierten Asthma $[4,17]$. Ursächlich können eine nicht hinreichend effektive Implementierung, Umsetzungs- bzw. Adhärenzbarrieren auf Seiten der Patienten [18] oder ein komplexes Zusammenspiel mehrerer Faktoren sein [5]. In einer multimodalen Studie haben die Autoren dies exploriert: Im qualitativen ersten Teil der Studie wurde in Fokusgruppen (FG) mit Patienten und Hausärzten deren Qualitätsvorstellungen und Therapieziele sowie Leitlinienempfehlungen und Umsetzungsbarrieren thematisiert. Im zweiten, hier dargestellten Teil wurde das Wissen um Leitlinieninhalte bei Hausärzten und Ärzten in Weiterbildung zum Allgemeinarzt untersucht. Die Praxistauglichkeit der deutschsprachigen Asthmaleitlinien-Kurzfassung wurde im dritten, qualitativen Studienteil von Ärzten bewertet ( $\bullet$ Abb.1). 


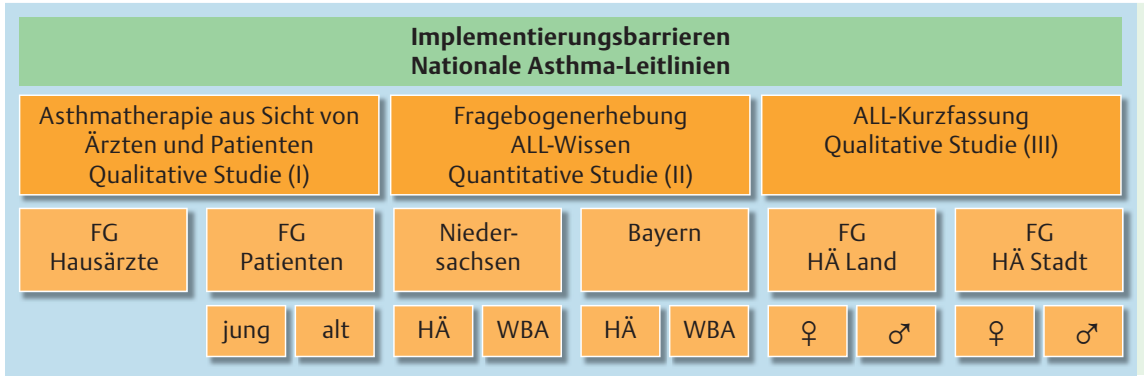

Abb. 1 Multimodaler Ansatz zur Eruierung von Implementierungsbarrieren (HÄ: Hausärzte; WBA: Weiterbildungsassistenten).
Da die inhaltliche Kenntnis von Leitlinien einer der ersten und wichtigsten Schritte für deren Anwendung ist, wird hier zunächst auf die Studie zum Leitlinienwissen eingegangen und diese mit ähnlichen nationalen und internationalen Studien verglichen $[19,20,21]$. Über die kassenärztlichen Vereinigungen wurden alle in hausärztlichen Praxen tätigen Weiterbildungsassistenten (WBA) in Niedersachsen und Bayern kontaktiert und um Teilnahme gebeten (45\% Responder). Zum Vergleich wurde eine Convenience-Stichprobe von Hausärzten in Niedersachsen im Rahmen von Qualitätszirkeln, in Bayern auf einer universitären Fortbildungsveranstaltung für Allgemeinmedizin rekrutiert. Der verwendete Wissensfragebogen „TAB-Quiz 15+3“ wurde aus dem Fragenpool verschiedener nationaler und internationaler Instrumente zusammengestellt, pilotiert und mit Experten der Deutschen Atemwegsliga konsentiert. Er enthielt 15 geschlossene Einzelfragen sowie 3 Fallvignetten mit je 2 Fragen. In allen Fragen konnten mehrere Antwortoptionen richtig sein. Für richtig angekreuzte Antwortmöglichkeiten gab es je einen Punkt, für falsch angekreuzte oder fälschlich nicht markierte korrekte Antwortmöglichkeiten jeweils einen Minuspunkt. Die Fragebogen wurden deskriptiv ausgewertet, Unterschiede zwischen Hausärzten und WBA mit einfachen statistischen Verfahren (Chi², T-test, Mann-Whitney-U) geprüft, Unterschiede im Hinblick auf demografische Charakteristika mit einer Varianzanalyse (ANOVA).

\section{Studienergebnisse und Diskussion \\ $\nabla$}

An der eigenen Studie nahmen insgesamt 295 Ärzte teil. Davon erreichten die Hausärzte $(n=55)$ durchschnittlich $58 \%$, und die WBA ( $n=240) 57 \%$ der maximalen Punktzahl. Ein ähnlicher Fragebogen wurde in der 2006-2008 in Nordrhein-Westfalen durchgeführten Leitlinien-Implementierungs-Studie Asthma (LISA) verwendet. Im Vorfeld einer Interventionsstudie wurden dort 263 Hausärzten je 4 Fragen zum üblichen Asthmamanagement und 13 Fragen zum leitlinienkonformen Wissen gestellt [20]. Bei den 4 LISA-Fragen zum üblichen Vorgehen in der eigenen Praxis erreichten 86,7\% der Befragten zwischen 76 und $100 \%$ der maximalen Punktzahl (nur 0,8\% erreichten weniger als 50\%). Abb. 2 vergleicht, wie viele Ärzte bei der eigenen Studie und der LISA-Wissensbefragung welchen Anteil der jeweils maximalen Punktzahl erzielten.

In internationalen Studien finden sich ähnliche Ergebnisse: In einer amerikanischen Studie wurden 720 Fachärzte und Ärzte in Weiterbildung für Innere Medizin vor und nach einem OnlineAsthma-Weiterbildungsmodul einem Multiple-Choice-Test zu den Themen Pathophysiologie, Diagnostik und Management des Asthmas unterzogen. Vor der Fortbildung wurden im Durchschnitt 54,2\% der maximalen Punktzahl erreicht, danach 75,6\% [19]. Einen asthmabezogenen Wissensfragebogen auf der Webseite der britischen UK General Practice Airways Group beant-

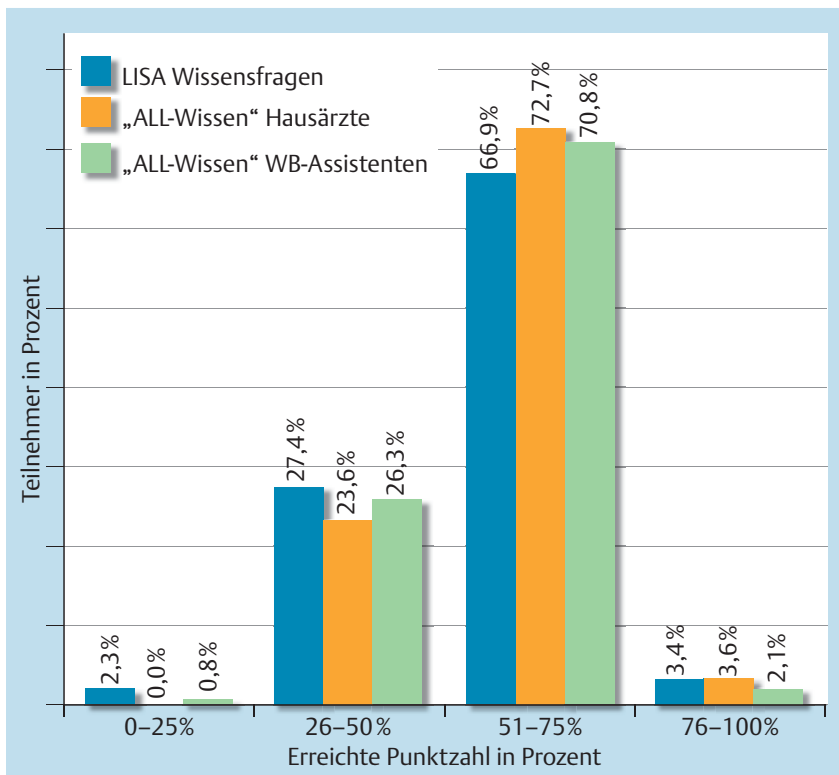

Abb.2 Vergleich von LISA und des eigenen TAB-Quiz 15-3.

worteten 96 Allgemeinärzte, die im Mittel 42\% richtige Antworten erzielten [21].

\section{Inhaltliche Stärken und Schwächen \\ $\nabla$}

Schwächen zeigten die nordrhein-westfälischen Hausärzte (LISA) vor allem in der Schweregradeinteilung, der Notfalltherapie und der Patientenschulung (genauere Angaben werden nicht gemacht) [20]. Die niedersächsischen und bayerischen Ärzte unserer Studie hatten größere Wissenslücken in den Themengebieten

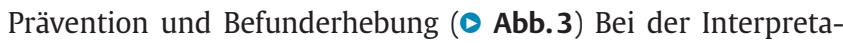
tion des Vergleichs muss berücksichtigt werden, dass in den beiden Studien unterschiedliche Fragebogen mit unterschiedlich vielen Fragen zu einem Themengebiet verwendet wurden, sodass die unterschiedlichen Themengebiete nicht in gleicher Weise abgebildet waren.

\section{Deutliche Wissensmängel in Teilbereichen $\nabla$}

Obwohl die chronisch entzündliche Genese des Asthmas lange bekannt ist, wussten knapp $40 \%$ der befragten Hausärzte und WBA dies nicht, vergleichbar den Ergebnissen einer pakistanischen Studie [22]. Zu anamnestischen Angaben und möglichen Befunden erreichten die Ärzte im Schnitt nur 2,2 von 6 Punkten (37\% richtige Antworten). Viele erkannten nicht, dass man bei Brustenge, Luftnot in staubiger Umgebung und einem leisen 


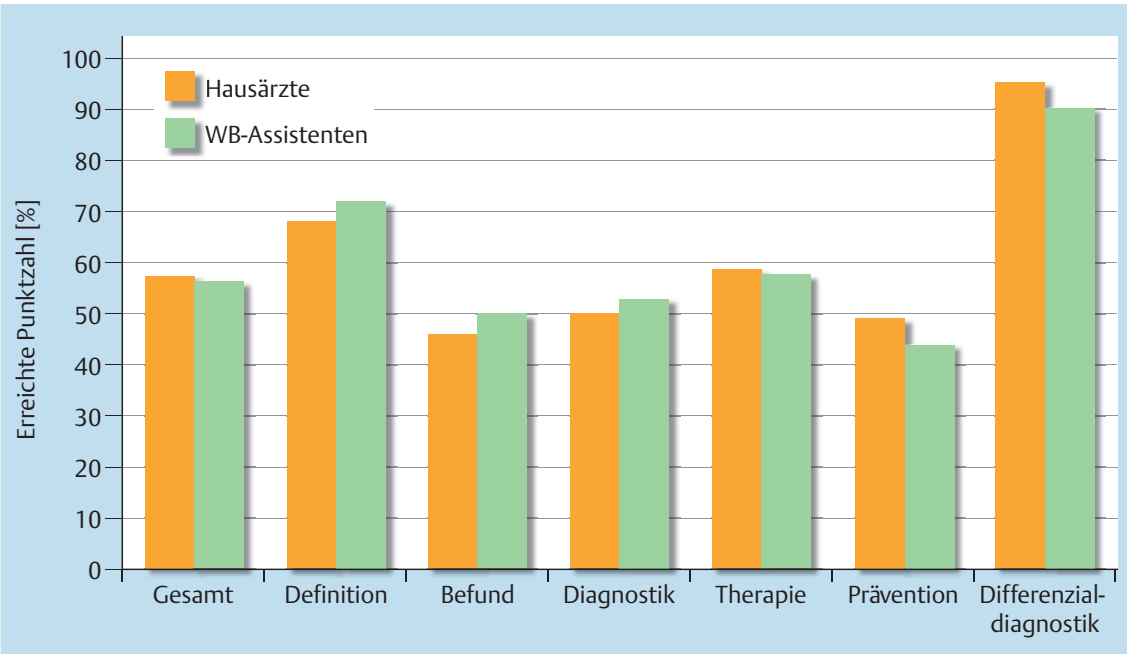

Abb.3 Ergebnisse der TAB-Quiz 15+3-Wissensbefragung bezogen auf Themengebiete oder sogar fehlendem Atemgeräusch an das Vorliegen eines Asthmas denken sollte. Die Symptome eines sehr schweren Asthmaanfalls konnten die WBA mit 62\% der zu erreichenden Punkte tendenziell etwas besser erkennen als die Hausärzte mit 54\%, wobei der Unterschied nicht signifikant war. Die Durchführung eines Reversibilitätstests mit systemischen Kortikosteroiden zur Diagnosesicherung kannten 92\% der Hausärzte und WBA nicht. Die Behandlung mit Kortikosteroiden, in inhalativer oder systemischer Form, scheint noch mit Unsicherheit behaftet: So glaubten $18 \%$ der Weiterbildungsassistenten bzw. 26\% der Hausärzte, dass inhalative Kortikosteroide (ICS) erst ab Stufe 3 des Stufenplanes, also bei mittelgradig persistierendem Asthma, zu verschreiben sind. Immerhin 10\% dachten fälschlicherweise, dass ICS in der Langzeittherapie ein hohes Risiko für Nebenwirkungen mit sich bringen, und 62\% der WBA sowie 69\% der Hausärzte wussten nicht, dass bei einem leichten bis mittelschweren Anfall eine systemische Kortikosteroidgabe erfolgen sollte. Die Bereitschaft, Mukolytika und Antibiotika einzusetzen, missachtet die negative bzw. sehr restriktive Leitlinienempfehlung: 69\% der befragten Ärzte hielten Mukolytika für bedarfsweise indiziert; $60 \%$ würden einer Patientin mit bekanntem Asthma und zunehmender Bronchialobstruktion und Sputumbildung ein Antibiotikum verschreiben, obwohl in der beschriebenen Fallgeschichte klinisch kein Hinweis auf einen bakteriellen Atemwegsinfekt bestand (ausdrücklich: Bonchialobstruktion, Atemnot, vermehrtes Sputum, aber kein Schnupfen, kein Husten, kein Fieber). Die Leitlinien weisen ausdrücklich darauf hin, dass die meisten Atemwegsinfekte viral bedingt sind, Antibiotika aber nur „bei gleichzeitig bestehender Pneumonie oder bakterieller Infektion der oberen Atemwege“ eingesetzt werden sollen. Leider fehlt die Angabe konkreter Kriterien für eine Antibiotikaindikation bzw. der Hinweis darauf, dass eine Differenzierung anhand klinischer Zeichen schwierig ist. Mit $\beta_{2}$-Sympathomimetika sowie nichtmedikamentösen Maßnahmen wie Raucherentwöhnung, Gewichtsreduktion, Allergenkarenz und Patientenschulungen konnte der Großteil der befragten Ärzte gut umgehen. Insgesamt erreichten sie im Themengebiet Therapie durchschnittlich $58 \%$ der maximalen Punktzahl. Die Umsetzung einer leitliniengerechten Therapie beinhaltet auch die richtige Anleitung von Patienten. Nicht optimal umgesetzt wird offenbar die empfohlene Aushändigung eines schriftlichen Medikationsplans inklusive einer Anweisung zur selbständigen Änderung der Medikation im Falle einer Symptomverschlechterung: 58\% der Weiterbildungsassistenten und $42 \%$ der Hausärzte stimmten dieser Option nicht zu.
In anderen Studien wollten Ärzte nur zwischen 5\% bis 33\% ihrer Patienten einen schriftlichen Medikationsplan mitgeben $[20,23$, 24], z.T. erklärten bis zu $90 \%$ der befragten Ärzte, dass sie vor jeder Therapieänderung konsultiert werden wollen [23].

\section{Implikationen \\ $\nabla$}

Wissensbefragungen zum Thema Asthma, aber auch das Handeln im Praxisalltag bilden insgesamt und international konsistent keine ausreichende Leitlinienadhärenz $a b$, obwohl die Versorgung von Asthmapatienten zu den täglichen Aufgaben eines Hausarztes gehört. Manche Studien betrachten den Zeitmangel der Ärzte als Hauptgrund dafür [24,25]. Pakistanische Allgemeinärzte, die weniger als 10 Jahre approbiert waren, erreichten durchschnittlich mehr Punkte als Ärzte, die seit mindestens 20 Jahren ihre Approbation hatten [22]. In unserer Studie erzielten Hausärzte, die seit weniger als drei Jahren niedergelassen waren, ein um knapp $10 \%$ besseres Gesamtergebnis als solche, die mehr als 15 Jahre niedergelassen sind $(p=0,033)$. Möglicherweise haben jüngere Ärzte bereits im Studium die Anwendung von Leitlinien erlernt. Auch die Einstellung dazu scheint eine Rolle zu spielen: Schottische Ärzte vertraten die Meinung, dass Leitlinien vor allem für Berufsanfänger nützlich seien, im späteren Berufsleben dagegen aufgrund fehlender Flexibilität und Individualität in Bezug auf den einzelnen Patienten eher seltener angewendet würden [26]. Womöglich führt dies dazu, dass sich manche ältere Ärzte auch weniger mit Leitlinien-Updates oder neuen Empfehlungen auseinandersetzen. Ob dies für das Behandlungsergebnis nachteilig ist oder durch die größere klinische Erfahrung und intuitiv richtiges Handeln (wie in der LISA-Studie angedeutet [20]) wieder ausgeglichen wird, wäre zu untersuchen. Auch die Zusammenarbeit in einer Praxisgemeinschaft ist ein möglicher Einflussfaktor [27]. Laut De Jong et al. werden Asthmaleitlinien am ehesten von Hausärzten genutzt, die in einer Einzelpraxis arbeiten und somit bei Fragen oder Ungewissheiten keine Kollegen konsultieren können [14]. In unserer Studie hatte jedoch die Praxisform (Einzelpraxis, Gemeinschaftspraxis oder Medizinisches Versorgungszentrum) keinen Einfluss auf das Ergebnis. Die Resultate der deutschen Studien zeigen bzgl. des Wissens von Fachärzten und Ärzten in Weiterbildung für Allgemeinmedizin um den Inhalt aktueller Asthma-Leitlinien noch deutlichen Spielraum für Verbesserung. Allerdings zeigen internationale Wissensbefragungen bei Ärzten für Allgemeinmedizin, 
Innere Medizin oder Kinderheilkunde ähnliche Ergebnisse. Vergleichbar suboptimales Wissen fand sich auch zu anderen Leitlinienthemen, z.B. Hypertonie, Herzinsuffizienz und koronarer Herzerkrankung [28]. Es bedarf also weiterer Anstrengungen, effektive Implementierungsstrategien zu identifizieren und einzusetzen und die Praxistauglichkeit von Leitlinien zu optimieren $[5,29]$.

\section{Fazit für die Praxis}

- Die deutschen Asthmaleitlinien haben das Ziel, die Versorgungsqualität zu verbessern und Ärzte bei der adäquaten Behandlung von Asthmapatienten zu unterstützen.

- Die Implementierung der Leitlinieninhalte in das hausärztliche Handeln ist verbesserungsbedürftig. Bei der Kenntnis der Leitlinieninhalte besteht Optimierungspotenzial bei Hausärzten und Ärzten in Weiterbildung.

- Zu Implementierungsbarrieren bzw. Gründen für eine nicht erwartungsgemäße Umsetzung von Leitlinienempfehlungen besteht weiterer Forschungsbedarf.

- Die Kenntnis von Leitlinieninhalten als auch die Nutzung von Leitlinien sollte durch geeignete Maßnahmen gefördert werden, die sowohl junge Ärzte in Studium und Weiterbildung als auch erfahrene Hausärzte berücksichtigen.

\section{Interessenkonflikt}

Die Studie wurde durch eine Spende der Deutschen Atemwegsliga e.V. an die Medizinische Hochschule Hannover finanziert. C.-P. Criée, H. Worth und P. Kardos sind Vorstandsmitglieder der Deutschen Atemwegsliga e.V., sonstige Interessenskonflikte bestehen nicht. E. Hummers-Pradier, H. Lingner, D. Piepenschneider und T. Schleef geben an, dass kein Interessenkonflikt besteht.

\section{Institute}

${ }^{1}$ Institut für Allgemeinmedizin, Medizinische Hochschule Hannover ${ }^{2}$ Abt. für Pneumologie, Schlaf- und Beatmungsmedizin, Ev. Krankenhaus Göttingen-Weende gGmbH, Bovenden-Lenglern

${ }^{3}$ Gemeinschaftspraxis \& Zentrum Pneumologie Allergologie Schlafmedizin, Klinik Maingau vom Roten Kreuz, Frankfurt

${ }^{4}$ Medizinische Klinik I, Klinikum Fürth, Fürth

${ }^{5}$ Institut für Allgemeinmedizin, Universitätsmedizin Göttingen

\section{Literatur}

1 Masoli M, Fabian D, Holt S et al. The global burden of asthma: Executive summary of the GINA dissemination committee report. Allergy 2004; 59: $469-478$

2 Buhl R, Berdel D, Criée CP et al. Leitlinie zur Diagnostik und Therapie von Patienten mit Asthma. Pneumologie 2006; 60: 139-177

3 Bundesärztekammer. Kassenärztliche Bundesvereinigung. Arbeitsgemeinschaft der Wissenschaftlichen Medizinischen Fachgesellschaften. Nationale Versorgungsleitlinie Asthma, Version 1.3. 2. Auflage. Im Internet: www.versorgungsleitlinien.de/themen/asthma/pdf/nvl_asthma_lang.pdf; Stand: 2011

4 Vermeire PA, Rabe KF, Soriano JB et al. Asthma control and differences in management practices across seven European countries. Respir Med 2002; 96: $142-149$

5 Kopp I. Perspektiven der Leitlinienentwicklung und -implementation aus der Sicht der AWMF. Z Rheumatol 2010; 69: 298-304

6 Arbeitsgemeinschaft der Wissenschaftlichen Medizinischen Fachgesellschaften. Ärztliches Zentrum für Qualität in der Medizin, Hrsg. Deutsches Instrument zur methodischen Leitlinien-Bewertung (DELBI) AWMF und ÄZQ. Im Internet: www.leitlinien.de/leitlinienmethodik/leitlinienbewertung/delbi/delbi-dokumente; Stand: 2008
7 Geisler LS. Treatment of acute and chronic airway obstruction with bronchospasmolytics in general practice. Dtsch Med Wochenschr 1980; 105: 1189-1191

8 Wettengel R, Berdel D, Hofmann D et al. Asthma therapy in children and adults. Recommendations of the German Respiratory League of the German Society of Pneumology. Med Klin 1998; 93: 639-650

9 American National Heart. Lung and Blood Institute. World Health Organisation NHLBI/WHO Workshop Report. Global Strategy for Asthma Management and Prevention. NIH Publication No 02-3659, 011995

10 Kroegel C, Wirtz $H$. History of guidelines for the diagnosis and management of asthma: From opinion to control. Drugs 2009; 69: 1189 - 1204

11 British Thoracic Society. Scottish Intercollegiate Guidelines Network. British Guideline on the Management of Asthma. A national clinical guideline. London, Edinburgh: BTS, SIGN. Im Internet: www.brit-thoracic.org.uk/guidelines.aspx; Stand: 2012

12 National Heart, Lung and Blood Institute. British Thoracic Society. Expert Panel Report (EPR-3) Guidelines for the diagnosis and management of asthma, Full Report 2007. US Department of Health Human Services, National Institutes of Health, National Heart, Lung and Blood Institute. Im Internet: www.nhlbi.nih.gov/guidelines/asthma/; Stand: 2007

13 Global Initiative for Asthma (GINA). Global Strategy for Asthma Management and Prevention. Im Internet: http://www.ginasthma. org/Guidelines/guidelines-resources.html; Stand 2012

14 de Jong JD, Groenewegen PP, Spreeuwenberg P et al. Do guidelines create uniformity in medical practice? Soc Sci Med 2010; 70: 209-216

15 Partridge MR, Fabbri LM, Chung KF. Delivering effective asthma care how do we implement asthma guidelines? Eur Respir J 2000; 15 : 235 237

16 Grol R, Grimshaw J. From best evidence to best practice: effective implementation of change in patients' care. Lancet 2003; 362: 1225 1230

17 Hasford J, Uricher J, Tauscher $M$ et al. Persistence with asthma treatment is low in Germany especially for controller medication - a population based study of 483,051 patients. Allergy 2010; 65: 347 - 54

18 Foster JM, Smith L, Bosnic-Anticevich SZ et al. Identifying patient-specific beliefs and behaviours for conversations about adherence in asthma. Intern Med J 2012; 42: 136-144

19 Hemnes AR, Bertram A, Sisson SD. Impact of medical residency on knowledge of asthma. J Asthma 2009; 46: 36-40

20 Koneczny $N$, Redaèlli $M$, Schürer $C$ et al. Wie „leitliniennah“ ist die hausärztliche Asthma-Behandlung? Teilergebnisse der L.I.S.A.-Studie. 10. Jahrestagung Deutsches Netzwerk Evidenzbasierte Medizin. [Vortrag] Berlin: 05.03. 2009. Im Internet: www.ebm-netzwerk.de/ kongress/2009/praesentationen/v3.3_koneczny.pdf; Stand: 2009

21 Pinnock $H$, Holmes $S$, Levy ML et al. Knowledge of asthma guidelines: Results of a UK general practice airways group (GPIAG) web-based "test your knowledge" quiz. Prim Care Respir J 2009; 19: 180-184

22 Haque AS, Zubairi AB, Shiraz A et al. Asthma knowledge and approach among Pakistani family physicians and the impact of an educational programme. Int J Tuberc Lung Dis 2007; 11: 1260 - 1265

23 Braido F, Baiardini I, Menoni S et al. Asthma Management Failure: A Flaw in Physicians' Behavior or in Patients' Knowledge? J Asthma 2011; 48: $266-274$

24 Sun YH, Eun BW Sim SY et al. Poor adherence and reasons for nonadherence to the asthma guidelines among pedriatricians in Korea. Asian Pac J Allergy Immunol 2010; 28: 147 - 154

25 Butzlaff $M$, Vollmar HC, Floer B et al. Learning with computerized guidelines in general practice? A randomized controlled trial Fam Pract 2004; 21: $183-188$

26 Moffat M, Cleland J, van der Molen T et al. Poor communication may impair optimal asthma care: a qualitative study. Fam Pract 2007; 24: 65 70

27 Wiener-Ogilvie S, Huby G, Pinnock $H$ et al. Practice organisational characteristics can impact on compliance with the BTS/SIGN asthma guideline: qualitative comparative case study in primary care. BMC Fam Pract 2008; 9: 32

28 Karbach U, Schubert I, Hagemeister J et al. Physicians' knowledge of and compliance with guidelines: an exploratory study in cardiovascular diseases. Dtsch Arztebl Int 2011; 108: 61 - 69

29 Treweek S, Oxman AD, Alderson P et al. Developing and evaluating communication strategies to support informed decisions and practice based on evidence (DECIDE): protocol and preliminary results. Implement Sci 2013: epub ahead of print, doi: 10.1186/1748-5908-8-6 